American Journal of Applied Sciences 5 (3): 189-196, 2008

ISSN 1546-9239

(C) 2008 Science Publications

\title{
A Design Strategy for Volumetric Efficiency Improvement in a Multi-cylinder Stationary Diesel Engine and its Validity under Transient Engine Operation
}

\author{
${ }^{1}$ P. Seenikannan, ${ }^{2}$ V.M.Periasamy and ${ }^{3}$ P.Nagaraj \\ ${ }^{1}$ Deptartment of Mech., Sethu Institute of Tech., Kariapatti, T.N, India, 626106 \\ ${ }^{2}$ Crescent Engineering College, Chennai, T.N, India \\ ${ }^{3}$ Deptartmentof Mech, Mepco Schlenk Engg College, T.N., India
}

\begin{abstract}
This paper proposes an approach to improve engine performance of volumetric efficiency of a multi cylinder diesel engine. A computer simulation model is used to compare volumetric efficiency with instantaneous values. A baseline engine model is first correlated with measured volumetric efficiency data to establish confidence in the engine model's predictions. A derivative of the baseline model with exhaust manifold, is then subjected to a transient expedition simulating typical, in-service, maximum rates of engine speed change. Instantaneous volumetric efficiency, calculated over discrete engine cycles forming the sequence, is then compared with its steady speed equivalent at the corresponding speed. It is shown that the engine volumetric efficiency responds almost quasi-steadily under transient operation thus justifying the assumption of correlation between steady speed and transient data. The computer model is used to demonstrate the basic gas dynamic phenomena graphically. The paper provides a good example of the application of computer simulation techniques in providing answers to real engineering questions. In particular, the value of a comprehensive analysis of fundamental physical phenomena characterizing engine mass flow is demonstrated.
\end{abstract}

Key words: Multi-cylinder engine, engine modeling, volumetric efficiency, exhaust manifold, computer simulation

\section{INTRODUCTION}

Optimum engine cylinder charging is achieved by breathing of an engine dependent on the design of intake and exhaust systems ${ }^{[1]}$. Hall K.G. studied the Design optimization of engine induction systems with different optimization techniques and methodology ${ }^{[2]}$ Steady speed volumetric efficiency is widely used as a parameter to characterize engine mass flow, and is a simple measurement to take. It is generally assumed in engine management applications that steady speed data is also applicable to transient operation i.e. under changing speed and load. This is the so-called 'quasisteady assumption' and it is based on the premise that the engine's mass flow characteristic will pass through a sequence of steady speed states during a transient expedition ${ }^{[3]}$. The consequence to this assumption is that the gas flows in the engine system must adjust infinitely quickly to changes in boundary conditions. Smith L.A provides the good knowledge and example about the Engine breathing-steady speed volumetric efficiency and its validity under transient engine operation, specifically engine speed and shaft load ${ }^{[4]}$. However, gas inertia and compressibility and the constraints of the manifold geometry, govern the rate at which changes in gas state can actually take place. It takes a finite time to communicate the effects of boundary changes throughout the engine system, and intermediate gas states may not be adequately described by a quasi-steady model. Multidimensional modeling of cold flows and turbulence in reciprocating engines have been tried by David Gosman ${ }^{[5]}$.

A further important factor to consider with reciprocating engines is that the flow is highly unsteady, even at steady engine speed and load. This means that in a firm thermodynamic sense, a steady state equilibrium condition is never achieved. Reciprocating engines are, therefore, always operating under transient, i.e. time-varying, gas dynamic conditions. Transient heat transfer modeling in automotive exhaust systems is well described by Konstantinidis ${ }^{[6]}$.

At some estimated steady operating point, a dynamic residual standing pressure wave profile is established in the engine manifolds. This waveform cyclically repeats with a period that is some multiple of the engine cycle period, and this is usually unity. For the quasi-steady assumption to be valid the established

Corresponding Author: $\quad$ P. Seenikannan, Deptartment of Mech., Sethu Institute of Tech., Kariapatti, T.N, India, 626106 
dynamic waveform in the manifolds must move from state-to-state infinitely quickly. This paper uses an engine performance analysis tool, the Gamma Technologies GT-Power6.2 code ${ }^{[7]}$, to establish whether the quasi-steady assumption is a justifiable one in automotive applications or not. The study is limited to typical automotive four-stroke compression ignition engines operating under full open Throttle.

A baseline demonstration of the predictive correctness of the engine performance analysis tool is given for the case of a full open Throttle steady speed torque curve on a production six cylinder engine. This provides a measure of confidence in the correctness of the underlying flow physics models, and the numerical solution schemes adopted. Section 5 provides the details of the baseline induction system, which was placed with a manifold of some current and leading edge designs. This provided the opportunity to compare the transient response of the engine with different modes of operation of the y-section manifold and the design procedures are briefly described in the section 6 .

To ascertain realistic maximum rates of change of engine speed under full open throttle measured instationary engine data were obtained and the details are listed in section 7 . The rates were then applied as boundary condition data in the engine model. Section 8 describes the output of the transient runs and step change transient runs data. Volumetric efficiencies simulated respectively under steady speed and transient conditions were then compared over the transient excursions. Finally, the engine model was run for the limiting case of a step change in engine speed at full open Throttle to show graphically the rate at which gas dynamic state can adjust to a pathological change. This provided a perfect test of the validity of the quasisteady assumption for a realistic engine even if under limiting conditions. Possible result modes are given for steady speed full open throttle conditions and a discussion of the results follows in section 9 and concluding remarks are made in section 10 .

Engine volume flow rate measurement: Engine theoretical volume flow rate is calculated simply from the engine speed and the swept volume of the engine by Speed Density Method. This method of calculating the mass flow rate is described in more detail elsewhere ${ }^{[8]}$. If an estimate of the inlet manifold density is known from manifold air pressure and temperature measurement then the theoretical engine mass flow can be calculated. In practice, however, the engine is not a perfect pump and the theoretical volume flow rate must be scaled by the volumetric efficiency. The Speed-
Density Method remains popular as a means of calculating engine air mass flow but is alone in requiring detailed knowledge of the engine's volumetric efficiency characteristic. Tillock provides the mass flow measuring techniques in the study paper Measurement and modeling of thermal flows in an air-cooled engine ${ }^{[9]}$.

Cylinder scavenge effectiveness and volumetric efficiency: The volumetric efficiency is the parameter describing the effectiveness of the induction process. The volumetric efficiency is a parameter used to characterize engine system breathing per second. It is defined as the ratio of the actual volume flow per cycle to a theoretical maximum value based on engine swept volume. Engine breathing-steady speed volumetric efficiency and its validity under transient engine operation are deeply illustrated by $\mathrm{Smith}^{[4]}$.

The volume flows must share a common reference density. Equivalently, the volumetric efficiency can also be defined as the ratio of the actual mass flow per cycle to the mass calculated from the product of the swept volume and an arbitrary reference density. For engine mapping applications, the reference density is usually based on the inlet manifold plenum conditions, but this is more a matter of convenience than necessity. Many factors influence the volumetric efficiency. Reciprocating four-stroke engines are essentially positive displacement pumps. The efficiency of cylinder charging is a function of the engine rotational speed (time available to fill cylinder), and the pressure ratio time history across the cylinder poppet valves. Inlet manifold geometry, via excitation of tuning phenomena and the imposition of pressure loss, can obviously influence pressure upstream of the poppet inlet valves. Valve timing too is another important factor. Even exhaust system design detail can impact on the volumetric efficiency, by restricting or assisting the net flow through the engine. It is important to emphasize that the volumetric efficiency is not a measure of cylinder scavenge effectiveness or of mass trapping efficiency since flows can escape through the exhaust valve during overlap without necessarily mixing in-cylinder. It is a simple measure of engine breathing efficiency and flow resistance through the system.

Engine modelling - computational simulation techniques: This segment discusses the computational simulation solution techniques adopted in engine simulation software generally, and in the GT-Power6.2 code in particular ${ }^{[10]}$. Meisner $S$. has given the general 
features and over view of Computer simulation of intake and exhaust manifold flow and heat transfer. The most versatile method of the Development of prediction technology of Intake and exhaust system performance using computer simulation was adopted for the design by Ohnishi ${ }^{[11]}$.

The nature of the present study requires that some care be taken to ensure that the predictions of transient gas dynamic behaviour are physically meaningful. The conclusions drawn from this study are based on computer simulation. At the same time as the basic gas dynamic equation sets being solved have not changed over the past 20 to 30 years, the numerical techniques being employed to solve them have highly developed significantly.

GT-Power6.2 is one of many engine modeling tools marketed on a commercial basis. Such codes share a common assumption for modeling unsteady compressible flows in the engine intake and exhaust system i.e. a one-dimensional simplification of the flow governing equations of mass, momentum and energy conservation. Until recently, the most common solution techniques used by both industry and the academic institutions were based upon the numerical solution of the Method of Characteristics as proposed by Benson ${ }^{[12]}$ in 1964. Although these algorithms were sufficiently accurate for most practical purposes, they carried a number of limitations within their formulation. The primary drawback was the non-conservative implementation of the source terms in the 1-D equation set. This meant that geometrical discontinuities such as sudden enlargements and contractions had to be handled using iterative boundary solutions that were computationally time-consuming. Also the first-order quasi-linear solution of the characteristic equations caused unavoidable smearing of pressure and contact discontinuities. A major breakthrough in solution accuracy and computational efficiency was application of finite difference/finite volume solutions for 1-D unsteady flow. Authors such as Meisner ${ }^{[13]}$, Chapman ${ }^{[14]}$ present methods which apply second order accuracy differencing techniques whereby the solution at each time level is obtained from a combination of initial values at two time levels.

The 1-D compressible flow solution in GTPower6.2 is based on the work of Gosman ${ }^{[15]}$ developed for 3-D CFD applications, reduced to one dimension. The whole engine intake and exhaust system is divided into many small sub-volumes over which an explicitly conservative finite volume solution of mass and energy is applied. The momentum equation is applied on a 'staggered mesh' defined by the computational cell mid- points. This means that the cell to cell fluxes of mass, momentum and energy are defined at the cell boundaries and the fluid state update from one time level to another is provided at the cell midpoints. Rakopoulos C.D. Studied heat flow in the combustion chamber components under transient conditions using finite element analysis. Also he studied the behaviour of diesel engine in transient temperature fields. The differencing method applied is wholly or partly second order as the cell to cell fluxes are increasingly distorted in the velocity direction with increasing density gradients. This leads to a progressive application of a diffusive term with increasing density gradient to contain the possibility of numerically generated oscillations. As is common with such techniques, the time-step is governed by stability constraints. A powerful aspect of GT-Power6.2 is the finite volume approach applied to junctions within the system. The method caters for an expansion or contraction into the junction, flow bend angle, wall friction and heat transfer by combining mass and energy conservation with assistance from experimental data. These junctions may be 'stacked' to form a quasi-3D approximation of the complex geometries found in exhaust silencer assemblies and air cleaner boxes. The momentum equation is applied in a similar manner to the 'staggered mesh' representation for duct calculations. The authors are satisfied that GT-Power6.2 combines 1-D solution techniques with look-ups of empirical data to provide the best currently available compromise between computational speed and solution accuracy for engine design applications.

Baseline engine model - correlation with measurement: A GT-Power6.2 model of a production 6.075 liters diesel was developed. In baseline form this model was used to establish confidence in the predictive capabilities of the software. A second engine model, derived from this baseline, was used in the studies of transient volumetric efficiency with y-section exhaust manifold reported later. Figure 1 shows a schematic layout of the engine flow system.

Discrete modeling elements in the figure correspond to those used in the GT-Power6.2 model. The 6 cylinder diesel engine has two valves per cylinder and a simple induction system to each cylinder which are bounded by an upstream plenum chamber. A large air cleaner volume with entry diffuser is fitted. The engine is port fuel injected. On the exhaust side the exhaust manifolds connect all the cylinders with individual outlet pipes from the port and then made into a single pipe as outlet runner pipe. This is standard 


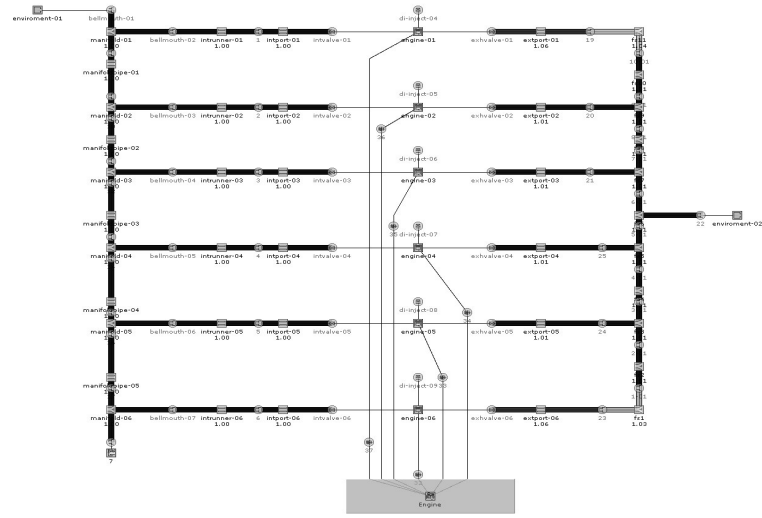

Fig. 1: Baseline engine model with existing manifold

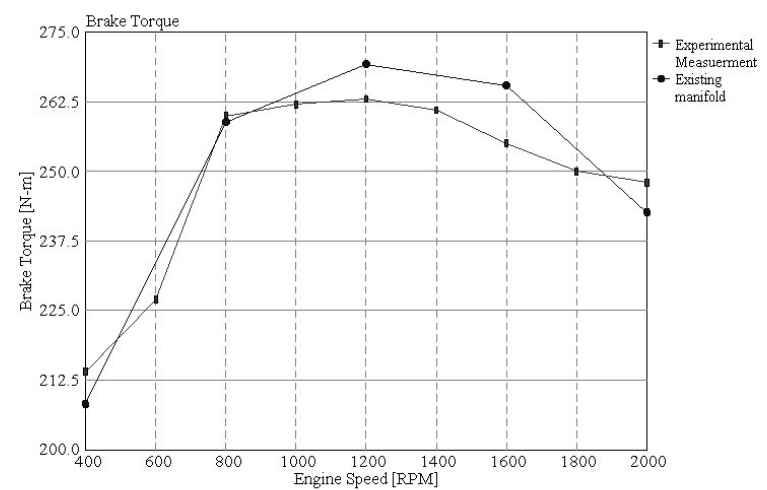

Fig. 2: Engine speed Vs brake torque

practice on 6 cylinder diesel engines. In modeling the engine much empiricism is required and many assumptions are often made. These assumptions are briefly described in the reference made by $\mathrm{Smith}^{[4]}$. Correlation with experimental data was limited to the full load line only. The measured data was obtained on an engine test bed.

Figure 2 shows a comparison of predicted full-load brake torque (circle points) with measured values (square points). The predicted line is plotted from simulation data with a resolution of $200 \mathrm{rpm}$. It is apparent that the simulated curve reproduces very closely the shape of the measured data. The process of adjusting model constants is equivalent to 'calibrating' the model. Frictional losses and dynamic valve timing are generally not known a standard one, hence the need to match initially measured and experimental values. What is important is that the correct basic shape of the torque curve is predicted, and this can be seen to be the case in Fig. 2. This usually implies that the volumetric efficiency, or engine breathing, characteristic has been correctly modeled.

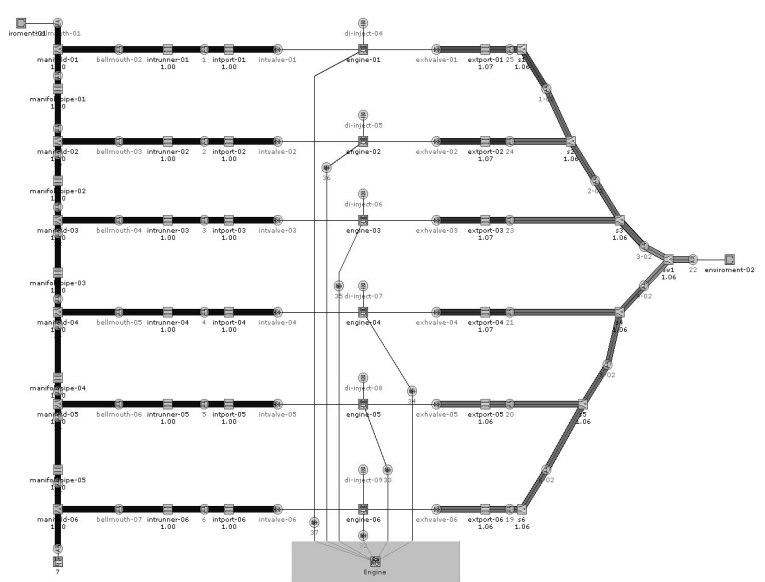

Fig. 3: Baseline Engine model with y-section manifold

Y-section manifold model: The baseline model provided a realistic prediction of the six cylinder engine's performance attention was confirmed and then the focus, turned to the main purpose of the study. The comparison of steady speed and transient volumetric efficiencies were obtained from the simulation code.

Factors likely to have an impact on transient response are inlet manifold volume and wave traversal distances within the manifold and also exhaust manifold volume. In recent years optimized design of inlet manifolds have been widely used to improve lowspeed torque characteristics ${ }^{[16,17]}$. Figure 3 shows a derivative of the baseline engine model in which the exhaust manifold is replaced by a y-section manifold.

The existing exhaust manifold (Fig. 1) is made up of six short bell mouth shaped out take runners connected to a large single outlet exhaust pipe.

The modified exhaust manifold system layout is made up of six primary pipes with $5 \mathrm{Y}$ junctions, the first connecting the $1^{\text {st }}$ and $2^{\text {nd }}$ exhaust runners, the second connecting. Output of $1^{\text {st }}, 2^{\text {nd }}$ and $3^{\text {rd }}$ runners the third connecting. $5^{\text {th }}$ and $6^{\text {th }}$ runners, the fourth connecting output of $5^{\text {th }}, 6^{\text {th }}$ and $4^{\text {th }}$ runners, the fifth connecting output of $1^{\text {st }}, 2^{\text {nd }}, 3^{\text {rd }}$ and output of $4^{\text {th }}, 5^{\text {th }}, 6^{\text {th }}$ after which they are connected to a muffler, which in turn connected to the atmospheric by means of a silencer ${ }^{[18]}$. Experimental and theoretical analysis of the flow in exhaust pipe junctions are briefly described in the analysis work by Flamang ${ }^{[19]}$. Kandylas I.P. relates Engine Exhaust system design based on heat transfer computation for the optimized design values of manifolds $^{[20]}$.

Measured in stationary engine data: The engine effectively operates with two separate exhaust systems, 


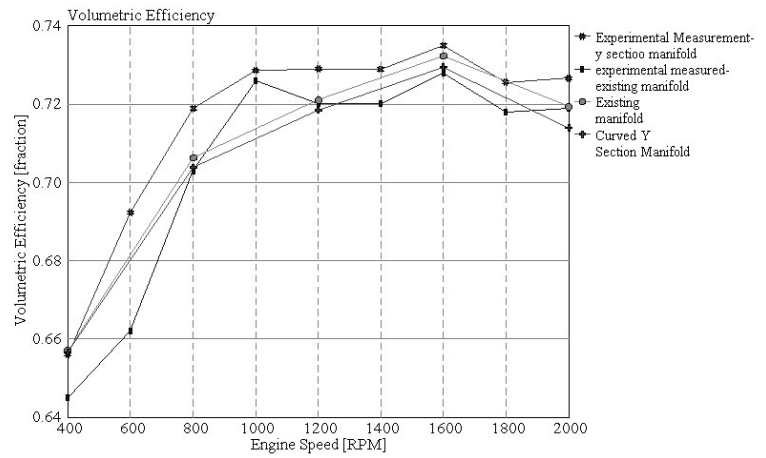

Fig. 4: Engine speed vs volumetric efficiency

with six cylinders, and comprising exhaust runners .The effect of introducing variation in the manifold geometry is to change fundamentally the pressure-time history across the inlet valves, and hence the mass flow rate through them. This is so-called manifold 'tuning'. Study on the design of inlet and exhaust system of a stationary internal combustion engine has been evaluated in his study by Ugur Kesgin ${ }^{[21]}$. Increased flow of air-fuel mixture can lead to direct increases in brake torque. One-dimensional engine performance prediction tools like GT-Power6.2 are ideally suited to exploring quantitatively the tuning benefits of manifold geometry changes. Figure 4 shows the predicted volumetric efficiency characteristics for each of the two exhaust manifolds.

Each mode has a distinctive shape. By running the two different computer simulations between modes benefits in peak torque can be obtained while comparing the simulations. In practice the use of all these modes, as shown in the figure, would provide a very pleasant drivability characteristic.

Similarly experimental measurement Modes with different speeds of starting from 400 to 2000, would probably give the best overall Compromising results, however, it is important to note that the manifold geometry has been selected almost arbitrarily for the present study. Discretisation was made to optimize the design in the simulation modes, when running the simulation. Existing manifold and y- section manifold configurations were selected for the comparison of steady speed and transient volumetric efficiencies. The data plotted in Fig. 4 provides the basis for comparison of both measured and simulated modes. In order to establish typical, maximum, in-service rates of change of engine speed, measurements were taken on an instrumented six cylinder diesel engine. The engine in this research work was a typical automotive test engine unit and the same as that modeled in this study. Various

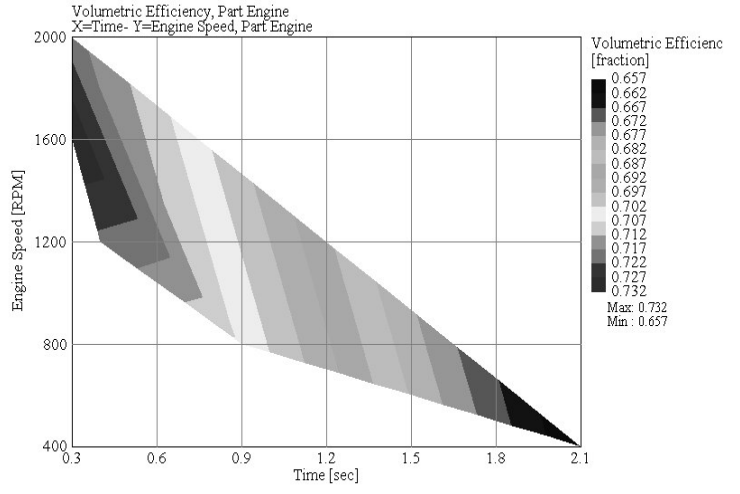

Fig. 5: Steady speed Vs transient volumetric efficiency (Existing manifold)

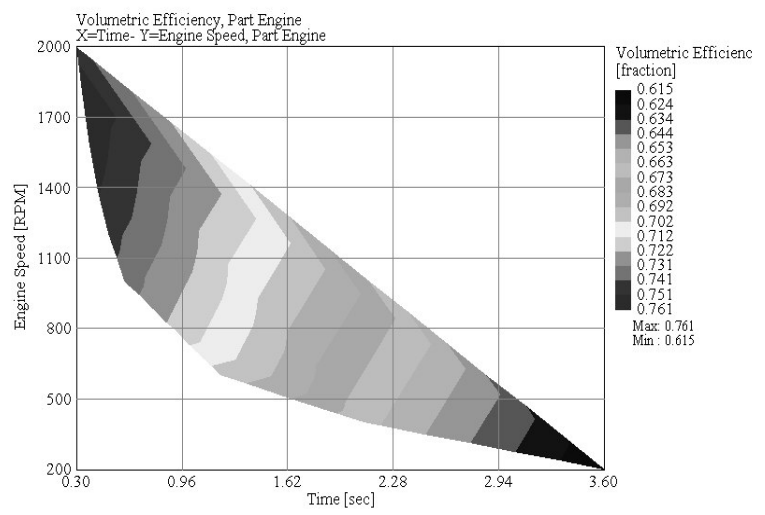

Fig. 6: Steady speed Vs transient volumetric efficiency (y-section manifold)

parameters were measured at high resolution during a various acceleration from a standing start.

Transient runs: Only the computational simulation manifold configurations were included for the transient runs. These two configurations correspond to two quite different cases, the first case was the existing manifold Mode and the second case was the y- section manifold Mode. Two transient runs in total were made, identical but for the position of the exhaust manifold system. The sequence commences with constant speed full load throttle operation at $1200 \mathrm{rpm}$ for a short period, is then followed by approximately 0.9 seconds of data with constant gradient, after which time the engine speed reaches $2000 \mathrm{rpm}$, and is then finally followed by a concluding interval of constant speed operation. With the run-time option used, the duration of the transient sequence is actually limited by the integer number of complete engine cycles specified by the user, which in this case were 50 . 
An excessive number of steady speed cycles were run simply to facilitate plotting of transient pressure profiles. Other transient boundary condition data were also required to be specified, including back pressure. In the steady speed runs measured back pressure data were used in the runtime case specification. For the transient runs an interpolated back pressure sequence corresponding to current engine speed was clearly specified. Similar provision was made for other speed dependent parameters which would become timevarying during the transients.

Figures 5 and 6 show the transient volumetric efficiency values plotted against their steady speed counterparts for two different exhaust manifolds. The separation of the data points is uneven because engine speed is changing along the time axis. Volumetric efficiency can only be calculated at the end of cycle hence the discrete nature of the data, and the use of data markers to indicate end of cycle. GT-Power6.2 calculates volumetric efficiency based on the mean engine speed during the engine cycle.

In addition to the volumetric efficiency plots a series plots of the pressure-crank angle histories at selected locations in the manifold was made. The results are for the Cylinder 1 exhaust runner, $45 \mathrm{~mm}$ from exhaust valve in Fig. 7 and also the results of the exhaust port in Fig. 8.

It is apparent from the results presented for the transient runs that the measured rate of change of engine speed, whilst typical of rapid acceleration, is not an extreme test of the quasi steady assumption. The definitive test would be to enforce a limiting rate of change of engine speed. In certainty, of course, engine inertia limits the rate at which speed can change. However, the simulation model permits the introduction of such a special case since the engine speed can be user defined or imposed.

Two step change transient runs were made. One for each of the existing manifold Mode and y-section manifold Mode of exhaust manifold cases. The transient imposed was an extreme one to accelerate engine speed from 400 to $2000 \mathrm{rpm}$ in a time interval of equivalent to one degree of crankshaft rotation at the mean speed of $1200 \mathrm{rpm}$. As in the earlier transient runs volumetric efficiency is plotted versus time with the equivalent steady speed values for each of the Mode cases.

\section{DISCUSSION}

The tests are principally based on the earlier work reported by $\mathrm{Smith}^{[4]}$. The inlet and exhaust manifolds

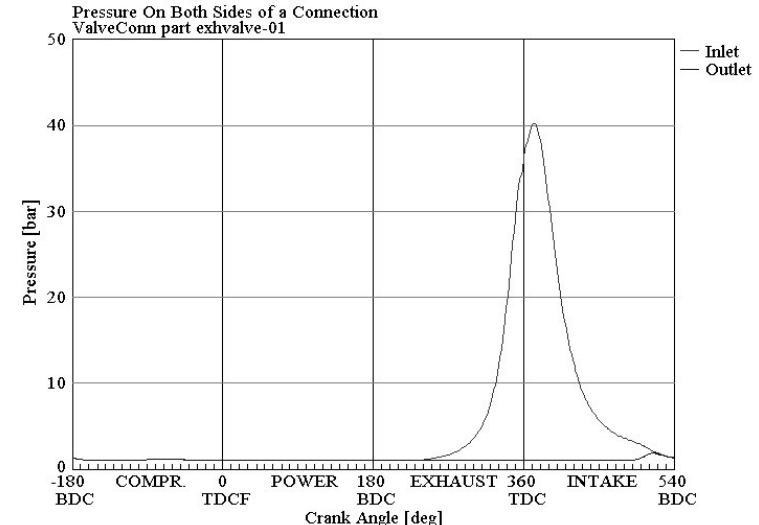

Fig. 7: Exhaust valve pressure (existing manifold)

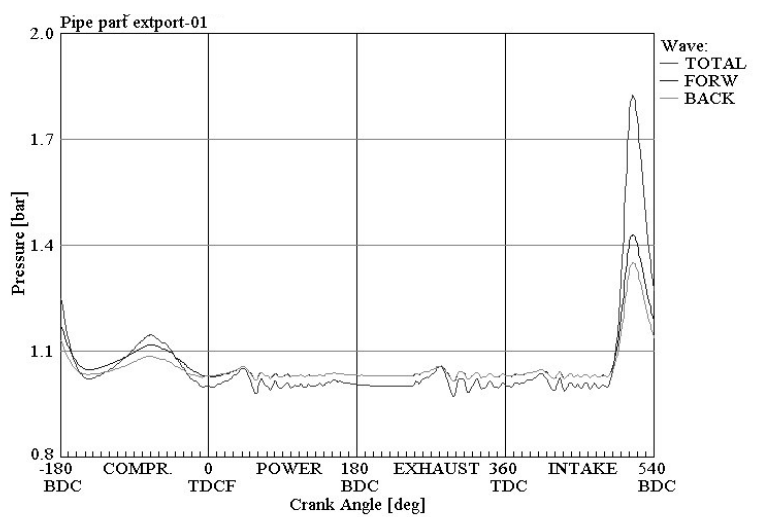

Fig. 8: Exhaust port pressure (existing manifold)

were included in the engine geometry to calculate flow through the intake and exhaust ports. The complete surface and edge features of the baseline engine manifolds were designed and imported from Pro-E package to the meshing tool GT-Power6.2 to generate the computer mesh. The prediction of the engine flow using a computer simulation code strongly depends on how accurately the initial and boundary conditions are specified. In GT-Power6.2, the simulation generally starts from the end environments for computational efficiency. It is customary to run the code for one complete cycle for obtaining reasonable initial conditions. The computational code was run for a number of cycles to obtain a converged initial condition. The solution is accepted as converged with the temperature.

The purpose of this study is to establish whether the quasi-steady assumption, as applied to engine volumetric efficiency, is a valid one. Acknowledged another way, the question is whether gas dynamic phenomena can adjust to rapid changes in boundary conditions such that, for all practical purpose, the 
engine mass flow is effectively the same as that at the corresponding steady engine. The simulation results presented in Fig. 5 and 6 compare transient and steady speed volumetric efficiencies under a realistic, inservice, rapid incline in engine speed, and at full load throttle. For the existing manifold case there is little or no distinct departure from steady speed volumetric efficiency values under transient operation, In the case of the y-section manifold a slight departure is apparent, initiated at the start of the transient, but steadily recovering as the transient progresses. These results clearly indicate that, at least for the case considered, the quasisteady assumption is a realistic one. This is an important result. It is creditable of note that this result could not easily have been obtained by experiment alone, the measurement of instantaneous engine mass flow rate is filled with difficulty. Engine volumetric efficiency is strongly controlled by exhaust runner pressure-time histories. Figure 7 shows a plot of crank angle based exhaust runner pressure at a location some $45 \mathrm{~mm}$ from the inlet valve, i.e. just upstream. In general, the transient parts of the sequence can be distinguished by non-stationary values at a given crank angle position in successive cycles. For steady speed operation such a section anywhere in the engine cycle will give a stationary value. This limiting case result, which confirms conclusively the validity of the quasisteady assumption for automotive IC engine applications, provides a good example of the power of numerical modeling techniques in answering real-world engineering questions.

\section{CONCLUSION}

Three important conclusions may be drawn from this study:

* Comparison between theoretical prediction and experimental measurements for the design of ysection configuration has been satisfactory, proving the validity of the developed model, while providing a sound foundation for engine performance comparisons of the model cases considered.

* The quasi-steady assumption, as applied to transient Automotive internal combustion engine volumetric efficiency under full load throttle conditions, is a valid one. Gas dynamic phenomena are capable of adjusting to even pathological rates of change of engine speed in a matter of a few engine cycles. For all practical purposes it is probably safe to assume response time scales of the order of milliseconds to even the most extreme of automotive in-service conditions.

* The computer simulation and analysis tools for engine performance analysis like GT Power6.2 provides an essential aid to the appraisal of engine performance of multi cylinder diesel engine. These advanced simulation tools authorize an analysis of engine processes at the most deep-seated level, providing valuable insight into the intricate causal inter-relationships characterizing internal combustion engine operation.

Future work: This study has measured compression ignition stationary diesel engine operation only. Subsequent modeling studies will consider for vehicle engine operation with or with out turbo charger, and the impact of exhaust manifold design strategies on transient response for both SI and turbocharged diesel applications.

\section{REFERENCES}

1. Phillip, B., C. Telford, D. Boam and J. Bingham, 2000. A design strategy for four cylinder SI automotive engine exhaust systems. J. SAE, 200001-0913.

2. Hall, K., 1989. Design optimization of engine induction systems. Autotech new engines, components and materials seminar, C399/20.

3. Heywood, J.B., 1988. Internal Combustion Engine Fundamentals. McGraw-Hill Book Co.

4. Smith, L.A., T. Fickenscher and R.P. Osborne, 1999. 'Engine breathing-steady speed volumetric efficiency and its validity under transient engine operation. SAE, 1999-01-0212.

5. David, G.A., 1985. Multidimensional modeling of cold flows and turbulence in reciprocating engines. SAE Paper 850344, pp: 111-127.

6. Konstantinidis, P.A., G.C. Koltsakis and A.M. Stamatelos, 1997. Transient heat tranafsr modeling in automotive exhaust systems. In: Proc. Inst Mech. Engineers, Part C: J. Mech. Engg. Sci., 211: $1-15$.

7. Gamma Technologies Inc., 2007. 'GT-POWER User's Manual'.

8. Zhaoda, Y., F. Zheng, Y. Xiaoli and S. Hongouan, 1988. 'Calculation and prediction of thermal loading of the air cooled Diesel engine. SAE Paper 881254.

9. Tillock, B.R. and J.K. Martin, 1996. Measurement and modeling of thermal flows in an air-cooled engine. Trans. SAE, J. Engines, 105: 1781. 
10. Galindo, J., J.M. Lujan, J.R. Serrano, V. Dolz and S. Guilain, 2004. Design of an exhaust manifold to improve transient performance of a high speed turbocharged diesel engine. Expl. Therm. Fluid Sci., 28: 863-875.

11. Ohnishi, K., H. Nobumoto, T. Ohsumi and M. Hitomi, 1998. Development of prediction technology of Intake and exhaust system performance using computer simulation. MAZDA Technical paper in Japanese, 6: 84-93.

12. Benson, R.S., R.D. Garg and D. Woollatt, 1964. A numeric solution of unstudy flow problems. Intl. J. Mech. Sci., 6: 114-117.

13. Meisner, S. and S.C. Sorenson, 1986. 'Computer simulation of intake and exhaust manifold flow and heat transfer. SAE Paper 860242.

14. Chapman, M., J.M. Novak and R.A. Stein, 1982. Numeric modeling of inlet and exhaust flows in multi-cylinder internal combustion engines. Flows in internal combustion engines, ASME WAM.

15. Gosman, A.D., 1985. Multidimensional modeling of cold flows and turbulence in Reciprocating engines. SAE Paper 850344.
16. Benajes, J., E. Reyes, J. Galindo and J. Peidro, 1997. Pre-design model for intake manifold in internal combustion engines. SAE paper 970055.

17. Heisler, H., 1995. Advanced engine technology. Chap. 5.

18. Badami, M., F. Millo and G. Giaffreda, 2002. Experimental and computational analysis of a high performance four-stroke motorcycle engine equipped with a variable geometry exhaust system. SAE Paper 2002-01-0001.

19. Flamang, P. and R. Sierens, 1989. 'Experimental and theoretical analysis of the flow in exhaust pipe junctions. ImechE, Paper No. VC382/082, pp: 461469.

20. Kandylas I.P. and AM. Stamatelos, 1999. 'Engine Exhaust system design based on heat transfer computation. Energy Conver. Manag., 40: 10571072.

21. Ugur, K., 2005. Study on the design of inlet and exhaust system of a stationary internal combustion engine. Energy Conver. Manag., 46: 2258-2287. 